\title{
Synthesis of Neodymium Hydroxide Nanoparticles with CTAB at Low Temperature
}

\author{
P. Martínez-Torres ${ }^{1}$, S. E. Borjas-García ${ }^{1}$, N. Gómez-Ortíz ${ }^{2}$, N. Dasgupta-Schubert ${ }^{3}$, J. L. Pichardo- \\ Molina $^{2}$ and D. R. García-Zavala ${ }^{4}$ \\ 1. Instituto de Física y Matemáticas, Universidad Michoacana de San Nicolás de Hidalgo, Morelia, \\ Michoacán, México. \\ 2. Centro de Investigaciones en Óptica A.C., León, Guanajuato, México. \\ 3. Facultad de Ciencias Físico-Matemáticas, Universidad Michoacana de San Nicolás de Hidalgo, \\ Morelia, Michoacán, México. \\ 4. Instituto Tecnológico de Morelia, Morelia, Michoacán, México.
}

Rare earths have been studied for multiple applications such as: photonic, luminescent material, thermoluminescent material, catalytic material and among others [1, 2]. Neodymium oxide is used as optical antireflection coating, gate insulators and protecting coatings. There are several methods to prepare neodymium oxide with different morphologies, such as sol-gel, hydrothermal treatment, spray pyrolysis, and microwave irradiation. Nanoparticles of neodymium hydroxide show an important value as a precursor material to obtain neodymium oxide. A typical procedure to obtain nanoparticles of neodymium hydroxide is using neodymium nitrate hexahydrate or alkoxides as precursors. However, there is not enough literature about the synthesis of neodymium hydroxide using neodymium chloride.

In this work, we synthesized neodymium hydroxide at low temperature using neodymium chloride hexahydrate (Aldrich), hexadecyltrimethyl ammonium bromide, CTAB (Sigma), sodium hydroxide (J. T. Baker) as Nd-source, organic surfactant and alkaline material, respectively. In a first step, two solutions were prepared. The first one was obtained by dissolving $1.7935 \mathrm{~g}$ of Nd-precursor and 1.8223 $\mathrm{g}$ of CTAB in $20 \mathrm{~g}$ of distilled water. For the second solution, $1.6 \mathrm{~g}$ of $\mathrm{NaOH}$ was dissolved in $10 \mathrm{~g}$ of distilled water. In a second step, the Na-solution was added slowly (drop by drop) to Nd-solution and stirred. The final solution was stirred and heat in a hot stir plate at $90{ }^{\circ} \mathrm{C}$ in order to evaporate the excess of water to get a material with a molar ratio of $\mathrm{Nd}$-source:CTAB: $\mathrm{NaOH}: \mathrm{H}_{2} \mathrm{O}$ equal to 1:1:8:150. The gel obtained was aged in a polypropylene bottle at $80{ }^{\circ} \mathrm{C}$ for 1 day. After the hydrothermal treatment, the sample was washed with $100 \mathrm{ml}$ of distilled water and centrifuged at $12000 \mathrm{rpm}$. After that, the material was dried at $80{ }^{\circ} \mathrm{C}$ for 1 day. On the other hand, it is necessary the calcination of the sample at $560{ }^{\circ} \mathrm{C}$ for $1 \mathrm{~h}$ to eliminate the residual organic surfactant.

The phase composition of specimen was analyzed by X-ray diffraction (XRD, D2 Phaser Bruker), with a Bragg-Brentano geometry and $\mathrm{Cu}-\mathrm{K} \alpha$ radiation $(\lambda=1.5418 \AA)$ and using the following scan: step size $=0.02^{\circ}, \mathrm{t}=5 \mathrm{~s}, 5^{\circ} \leq 2 \theta \leq 90^{\circ}$. The morphology of the as-prepared sample was analyzed using a high resolution scanning electron microscopy (HR-SEM, JEOL JSM-7800F). According to JCPDS 00070 0214 card, the phase of the material was identify as neodymium hydroxide, and the morphology corresponds to nanowires with diameters of around $15 \mathrm{~nm}$ and lengths of more than $100 \mathrm{~nm}$. This method seems to be easer than others procedures reported in the literature [3]. There are several works about the synthesis of nanorods of neodymium hydroxide where the hydrothermal treatment temperature is between 180 and $200^{\circ} \mathrm{C}$ [3]; in contrast, with our procedure, it was possible to obtain similar morphology at low hydrothermal treatment temperature $\left(80^{\circ} \mathrm{C}\right)$. One of the main applications of CTAB is as template to synthesize mesoporous metal oxide materials. However, in this procedure, we used 
CTAB to analyze its effect on the morphology during the synthesis of neodymium hydroxide nanoparticles [4].

\section{References:}

[1] M. Zawadzki and L. Kępiński, Journal of Alloys and Compounds 380 (2004) p. 255.

[2] W. Zhua et al, Materials Chemistry and Physics, 122 (2010) p. 362.

[3] N. Ekthammathat et al, Digest Journal of Nanomaterials and Biostructures, 10 (2015) p. 715.

[4] The authors acknowledge funding from H. Consejo Técnico of Instituto de Física y Matemáticas, and Consejo Nacional de Ciencia y Tecnología (CONACyT), México.

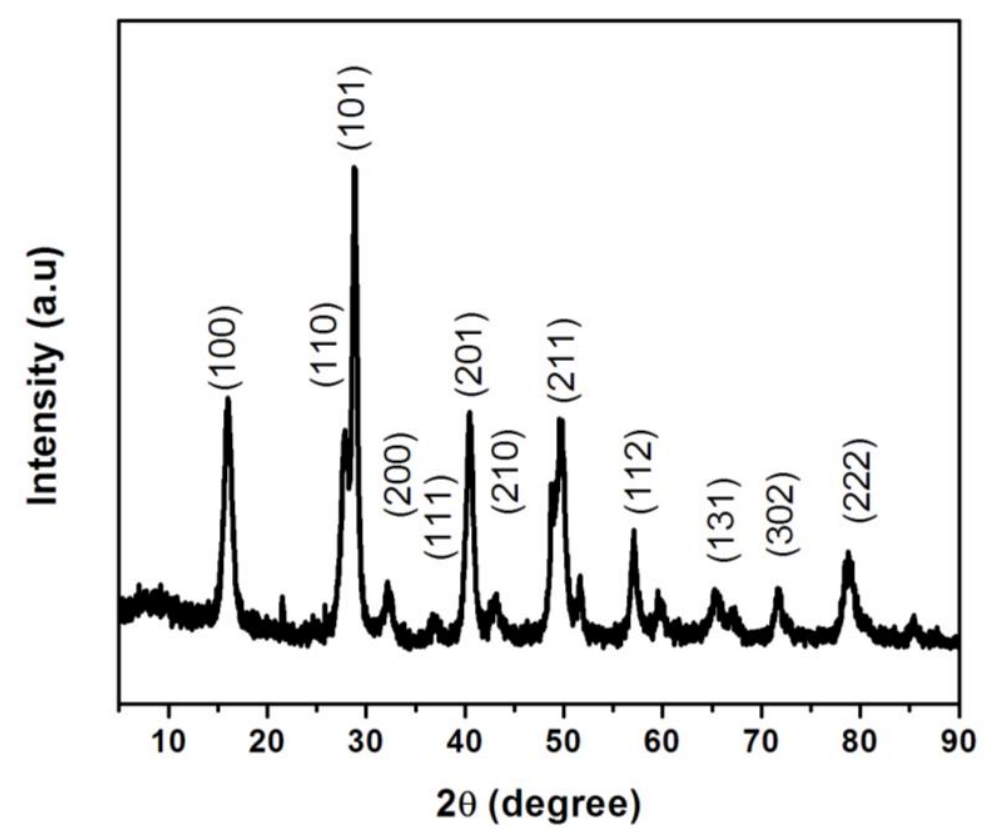

Figure 1. XRD pattern of neodymium hydroxide
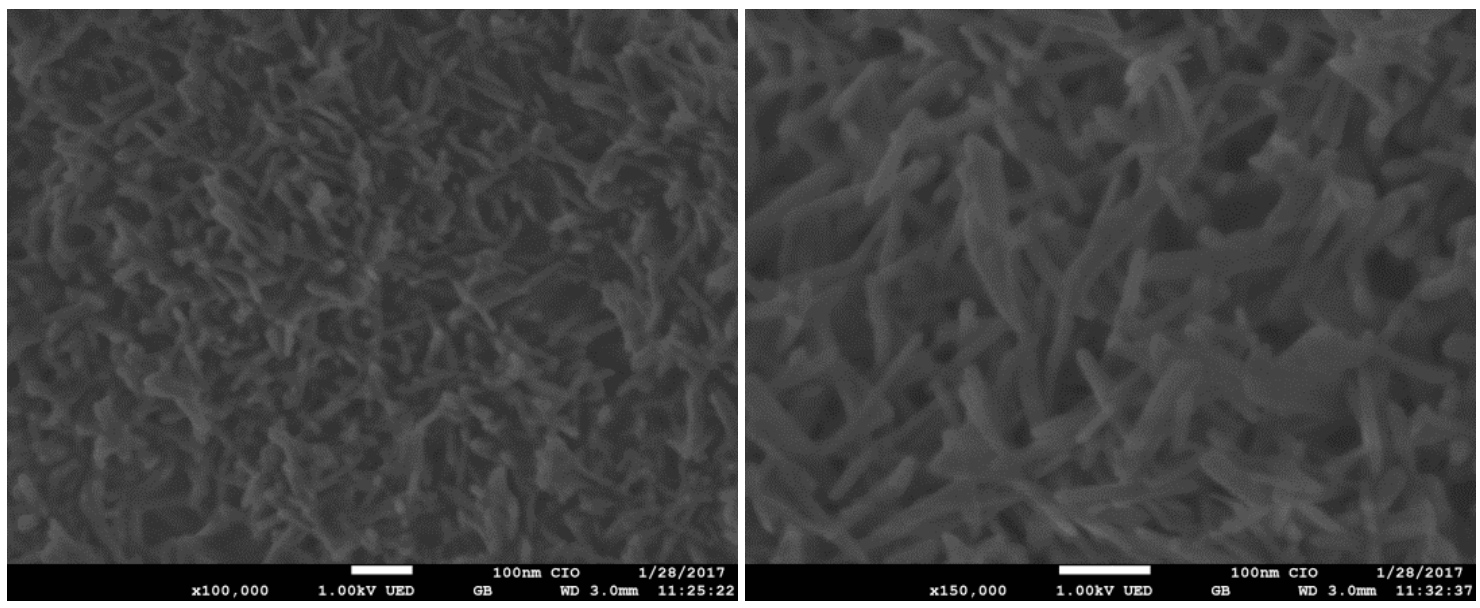

Figure 2. SEM images of neodymium hydroxide 